\title{
Distribuzione, consistenza ed evoluzione delle popolazioni di cigno reale, Cygnus olor, nidificanti in Italia nel periodo 1980-2012
}

\author{
Pierandrea Brichetti ${ }^{1 *}$, Nunzio Grattini ${ }^{2}$
}

\begin{abstract}
Riassunto - Storicamente, i primi casi di nidificazione di Cigno reale (Cygnus olor) in Italia sono stati accertati in Lombardia e Piemonte negli anni ' 60 -'70 del secolo scorso, in seguito ad introduzioni iniziate negli anni ' 30 -' 40 nelle parti svizzere di alcuni laghi lombardi e continuate nei decenni successivi. La popolazione nidificante risulta attualmente concentrata nelle regioni settentrionali, dal Piemonte orientale alla fascia costiera alto-adriatica, dove si riproduce quasi il $90 \%$ della popolazione nazionale; presenze più frammentate in Liguria, Trentino-Alto Adige, Toscana, Umbria, Lazio, Basilicata e Sardegna. All'inizio degli anni ' 80 del secolo scorso erano state stimate 20-35 coppie nidificanti in meno di 15 siti; di queste, 1' $80 \%$ si trovava in Piemonte e Lombardia. Le coppie sono aumentate a 25-45 nel 1987, poi a 300-500 nei primi anni del 2000 , ed attualmente a $500-700$ in oltre 150 siti, con un aumento di oltre il $900 \%$ rispetto ai valori iniziali. Questi dati confermano la fase di incremento ed espansione territoriale nota a livello europeo negli ultimi decenni. La colonizzazione di molti siti riproduttivi, localizzati in prevalenza nelle regioni settentrionali, è dovuta alla formazione di nuclei svernanti, cui fa seguito la formazione di coppie riproduttrici. Tale fenomeno ha interessato, seppur in misura più limitata, anche le regioni centro-meridionali, dove le osservazioni sono andate aumentando negli ultimi due decenni. Il Cigno reale nidifica a coppie isolate o localmente raggruppate in forma coloniale in zone umide naturali o artificiali, anche di modesta estensione, preferibilmente dal livello del mare fino a $300 \mathrm{~m}$, con un'altitudine massima di 970 m s.l.m., rilevata in Trentino.
\end{abstract} Italia.

Parole chiave: Cigno reale, distribuzione, consistenza, evoluzione,

Abstract: Distribution, abundance and trend of the breeding population of Mute Swan, Cygnus olor, in Italy in the years 1980-2012.

Historically, the first Mute Swan (Cygnus olor) nesting cases in Italy were found in Lombardy and Piedmont in the '60s-'70s of the last century, as a result of introductions started in the '30s-' 40 s in the Swiss parts of some lakes of Lombardy and continued in the following deca-

${ }^{1}$ CISO, Via V. Veneto 30, 25029 Verolavecchia (BS), Italia E-mail: pbrichetti@alice. it

${ }^{2}$ Gruppo Ricerche Avifauna Mantovano, Via P. Gobetti 29, 46020

Pegognaga (MN), Italia

E-mail: cristatus@virgilio.it

* Corresponding author: pbrichetti@alice. it

(C) 2014 Pierandrea Brichetti \& Nunzio Grattini

Received: $15^{\text {th }}$ October 2014

Accepted for publication: $19^{\text {th }}$ November 2014 des. At present, $90 \%$ of the breeding population of Mute Swan in Italy is located in the Northern regions, from Eastern Piedmont to the Northern Adriatic coast; smaller numbers are present in Liguria, TrentinoAlto Adige, Tuscany, Umbria, Lazio, Basilicata e Sardinia. In the early ' 80 s there were $20-35$ pairs in less than 15 sites, $80 \%$ of them in Piedmont and Lombardy; the numbers increased to $25-45$ in 1987, 300-500 in the early 2000s. At present, there are 300-700 pairs, in more than 150 sites, with a more than $900 \%$ increase. The colonization of many sites, mainly located in the northern regions, is due to the repeated presence of wintering birds, which was followed by the formation of breeding pairs; this phenomenon has also affected, although to a lesser extent, the central and southern regions, where observations in non-reproductive period have been increasing over the past two decades. These data confirm the number increase, and areal expansion, which have been recorded in Europe in the latest years. In Italy, Mute Swan nests in isolated pairs or locally grouped in natural or artificial wetlands, even of small size, preferably from sea level up to $300 \mathrm{~m}$, with a maximum altitude of $970 \mathrm{~m}$ asl found in Trentino.

Key words: Cygnus olor, distribution, abundance, trend, Italy.

\section{INTRODUZIONE}

Il Cigno reale, Cygnus olor, (Gmelin, 1789) è specie monotipica a distribuzione eurasiatica (Boano \& Brichetti, 1989), divenuta successivamente cosmopolita a seguito di introduzioni in Nord-America, Sud-Africa, Giappone, Australia e Nuova Zelanda. La popolazione europea è stimata in 54.000-69.000 coppie, con elevato incremento nel decennio 1990-2000 (BirdLife International, 2004) e presenze più consistenti nel Regno Unito, Svezia, Danimarca, Germania, Polonia e Olanda (Hagemeijer et al., 1997). Parzialmente migratrice, sedentaria e dispersiva. In Italia è sedentaria e nidificante, migratrice regolare e svernante (Brichetti \& Massa, 1998). Nella lista CISO-COI è considerata specie nidificante regolare che ha formato popolazioni in grado di autosostentarsi conseguenti a introduzioni da parte dell'uomo, fughe da cattività o alla presenza di individui giunti spontaneamente da popolazioni estere (Fracasso et al., 2009).

In tempi storici era ritenuta nidificante allo stato domestico o semi-domestico. I primi casi di nidificazione sono stati accertati in Lombardia e Piemonte negli anni '60-'70 del secolo scorso a seguito di introduzioni iniziate negli anni '30-'40 nelle parti svizzere di alcuni laghi 
lombardi e continuate nei decenni successivi. Introdotta nel Veneto alla fine degli anni '70, in Friuli nel 1981 e in Trentino negli anni '80; casi di nidificazione a seguito di immissioni anche in Emilia-Romagna, Toscana, Lazio e Sardegna; tentativo di riproduzione in Puglia a inizio anni '90.

All'inizio degli anni ' 80 stimate 20 -35 coppie di cui $1 ' 80 \%$ nidificanti in Piemonte orientale e Lombardia occidentale (Brichetti et al., 1984), aumentate a 25-45 coppie nel 1987 (Perco, 1988), passate a 300-500 coppie nei primi anni del XXI secolo, con tendenza all'incremento ed espansione territoriale (Brichetti \& Fracasso, 2003).

La distribuzione attuale, conseguente sia al consolidamento e all'espansione delle popolazioni naturalizzate, sia a successive introduzioni o fughe da cattività, risulta concentrata nelle regioni settentrionali, dal Piemonte orientale al Friuli-Venezia Giulia e nella fascia costiera alto-adriatica, tra le province di Trieste e Rimini, con presenze più frammentate in Emilia-Romagna interna e Lazio, molto localizzate in Liguria, Toscana, Umbria, Basilicata e Sardegna; da confermare in Puglia.

Il Cigno reale nidifica a coppie isolate o localmente raggruppate in forma coloniale in zone umide naturali o artificiali, anche di modesta estensione, con fondali poco profondi, ricche di vegetazione galleggiante e riparia emergente (laghi, fiumi, canali di risorgiva, cave, laghetti urbani, aree portuali, paludi, incolti umidi, casse di colmata, lagune e valli da pesca). Risulta più frequente dal livello del mare fino a $300 \mathrm{~m}$, con max. altitudinale di $970 \mathrm{~m}$ in Trentino nelle Paludi di Sternigo nella parte settentrionale del Lago di Serraia (Caldonazzi et al., 1997).

La specie in Europa è definita stabile e in evidente incremento negli ultimi decenni (BirdLife International, 2004). In Italia non era stata inserita nella Lista Rossa degli Uccelli nidificanti (LIPU \& WWF, 1999) e nell'attuale Lista Rossa è ritenuta Naturalizzata con una valutazione "Non Applicabile" (Peronace et al., 2012), mentre Brichetti \& Gariboldi (1992) hanno attribuito alla specie un "valore" nazionale di 50,8, abbastanza vicino a quello medio nazionale di 50,4.

Il presente lavoro ha lo scopo di definire l'attuale distribuzione e consistenza delle popolazioni nidificanti, valutandone l'evoluzione nel corso degli ultimi decenni a livello nazionale e regionale (Fig. 1).

\section{MATERIALI E METODI}

La raccolta dei dati è stata effettuata prevalentemente attraverso un'approfondita ricerca bibliografica, utilizzando la Banca Dati Ornitologica (Brichetti, 2012), integrata da numerose informazioni inedite avute da collaboratori. Nonostante ciò riteniamo che qualche sito riproduttivo possa essere sfuggito alla nostra indagine. Complessivamente si sono consultati 167 lavori pubblicati tra la fine del XIX secolo ed il 2011, apparsi su riviste nazionali, oltre che negli atti dei Convegni italiani di Ornitologia e sugli "Atlanti”.

\section{RISULTATI E DISCUSSIONE}

Vengono prese in considerazione ed elencate di seguito le sole regioni per quali la nidificazione è risultata certa o presunta tale nel periodo considerato (1980-2012).

Piemonte: in provincia di Novara una coppia nidificante dal 1980-85 sul Lago d'Orta; un'altra ha nidificato nel canneto di Fondo Toce sul Lago Maggiore nel 1971, con successive nidificazioni irregolari (Mingozzi et al., 1988) e nel 1991 (G.P.S.O., 1992); in provincia di $\mathrm{Cu}-$ neo localizzato lungo il basso corso del Tanaro e in bacini artificiali con individui sfuggiti alla cattività (Caula et al., 2005). A Torino 1-2 coppie nidificanti irregolarmente negli anni '80 e '90 (Maffei et al., 2001). In provincia di Vercelli nidifica dal 2009 lungo il Sesia (Bordignon \& Lonati, 2011); nel Verbano-Cusio-Ossola nidifica sui laghi Maggiore, di Mergozzo e d'Orta, sia in zone di canneto sia in ambienti altamente antropizzati come sul lungolago di Verbania (Bionda \& Bordignon, 2006); nella stessa provincia l'unico dato successivo all'atlante risale al giugno 2009 e riguarda l'osservazione di una coppia con un pullus in comune di Ornavasso (C. Fabrizio, com. pers.). Sul lago d'Orta una nidificazione in un nuovo quadrato (comune di Omegna), con una femmina in cova a fine aprile 2006, non confermata successivamente (C. Fabrizio, com. pers; Banca dati SSNVCO).

Liguria: un caso di nidificazione a Genova nel 1997 nelle Vasche del Parco pubblico dell'Acquasola (Borgo et al., 2005). Negli ultimi anni stimate a livello regionale 13-18 coppie, situate principalmente presso le foci dei torrenti: province di Imperia 6-7 coppie, Savona 4-5 coppie; Genova 2-4 coppie; La Spezia 1-2 coppie; la presenza della specie è dovuta essenzialmente ad introduzioni avvenute in passato per scopi ornamentali e si sostiene grazie al cibo fornito (R. Valfiorito, com. pers.).

Lombardia: nella regione stimate 15-25 coppie nel periodo 1983-87 (Brichetti \& Fasola, 1990) e successivamente 50-100 coppie (Vigorita \& Cucè, 2008). La popolazione dell'ultimo decennio può essere stimata in 140170 coppie. In provincia di Varese nidificante nel periodo 1983-87 nei canneti che bordano il Lago Maggiore (Angera e Monvalle) con segnalazioni di presenza anche nella parte settentrionale del lago Maggiore (foce del Tresa) e del lago di Lugano (Guenzani \& Saporetti, 1988), mentre nell'indagine del successivo atlante dei nidificanti (200305) la specie si è riprodotta sulle sponde del lago di Varese, Palude Brabbia e Lago di Comabbio. La popolazione provinciale nel periodo 1998-2000 era stimata intorno alle 40 coppie così ripartite: 15 sul Lago Maggiore, 12 sul Lago di Varese, 2-3 sul Lago di Comabbio, 2-3 sul Lago di Monate, 1-2 sul Fiume Tresa, 1-2 sul Fiume Ticino e 1 nella Palude Brabbia (Tosi \& Zilio, 2002), dove le coppie sono aumentate sino a 6 nel 2005, mentre nel 2006 non si è riprodotta nessuna coppia; questi dati sono abbastanza riconfermati nel periodo 2003-05 (Gagliardi et al., 2007). Nella parte del Lago di Como compreso nella provincia di Lecco, negli ultimi anni sono risultate presenti 4-6 coppie, sul Lago di Pusiano 3-4 coppie, sul Lago di Annone 2-3 coppie, sul Lago di Garlate 4-5 coppie, sul Lago di Olgi- 


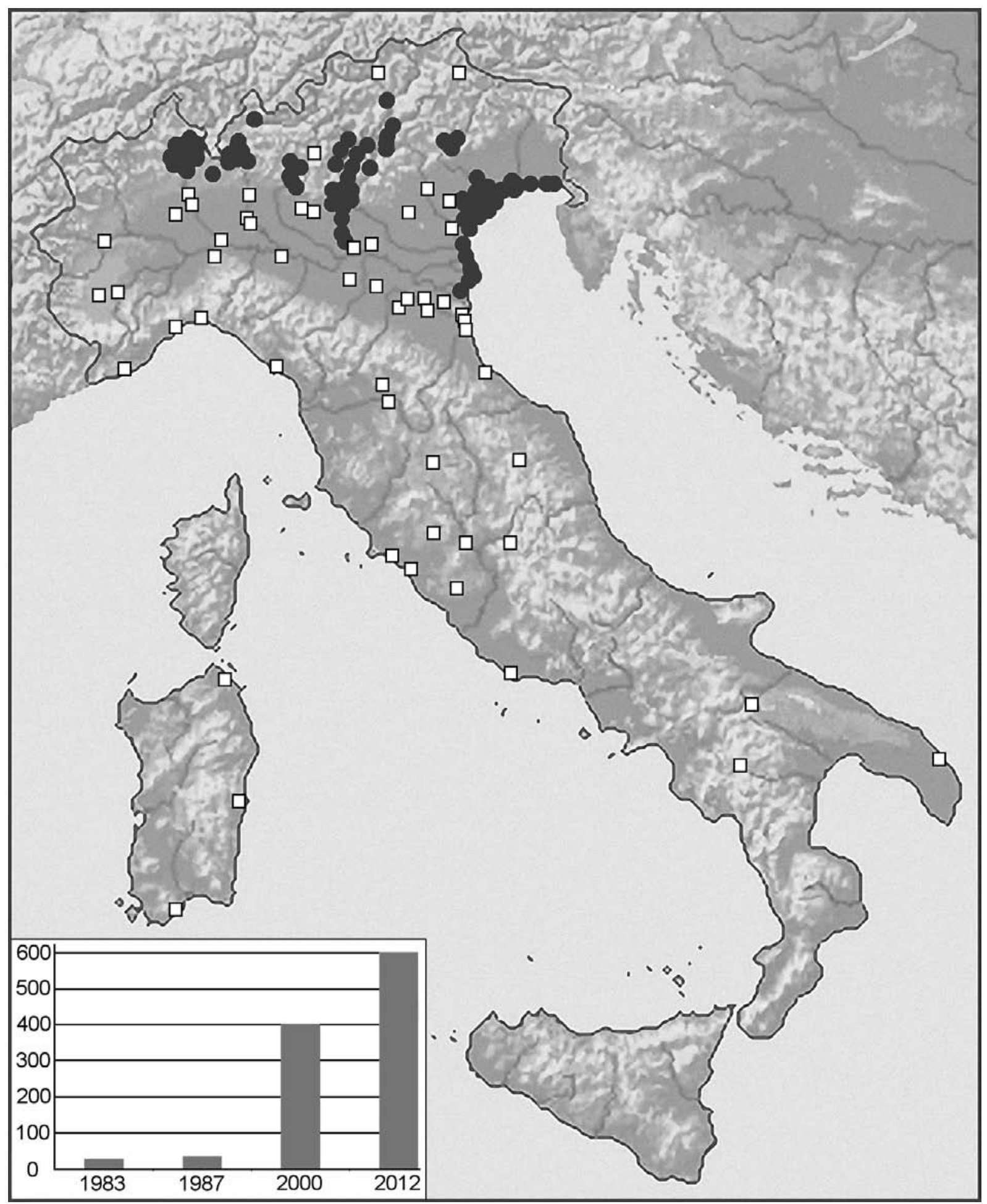

Fig. 1 - Areale attuale di nidificazione del Cigno reale (Cygnus olor) in Italia. Aree e tondi neri: siti riproduttivi consolidati dagli anni '80 (laghi insubrici, zone costiere friulane e venete) o colonizzati successivamente in seguito ad espansioni dei nuclei principali o conseguenti alla precedente formazione di nuclei svernanti. Quadrati bianchi: siti di presenza più recente dovuta sia a colonizzazioni spontanee da aree prossime (regioni settentrionali) sia, in buona parte, a locali introduzioni (regioni centrali, meridionali e Sardegna); il dato pugliese (Salento) riguarda un tentativo di nidificazione. Nel box: andamento della popolazione nidificante (numero medio di coppie) negli anni considerati. / Recent breeding distribution of the Mute Swan (Cygnus olor) in Italy. Black areas and dots: breeding sites occupied by the $80 \mathrm{~s}$ (Insubric lakes, coastal areas of Friuli and Veneto) or colonized by subsequent expansions of the core areas or resulting from the previous formation of wintering groups of birds. White squares: sites of the most recent presence due to spontaneous settlements from nearby areas (northern regions) or, in great part, due to local introductions (central-southern regions, Sardinia); data from Puglia (Salento) concerns an attempt to nest. In the box: Trend of the breeding population (average number of pairs) in the years considered. 
nate 2 coppie e sul Fiume Adda, tra Olginate a Paderno, 8-10 coppie; in alcune di queste e di altre località è presente un numero ben più elevato di individui non nidificanti, come circa 30-40 nel porticciolo di Lecco, 30 sul lago di Olginate e 20-25 sul fiume Adda in località Toffo comune di Brivio (E. Vigano, com. pers.). Nella parte settentrionale del Lago di Como singole coppie nidificanti nel 2011 e 2012 in località Dascio (Sorico), lungo il fiume Mera (Gera Lario) nel 2011 e una coppia nel 2012 sul Lago di Piano (M. Carabella, com. pers.). Sul Lago di Novate-Mezzola (CO-SO) nidificante dagli anni '70-'80 con 2-3 coppie, con max. di 7 a metà del primo decennio (Brichetti et al., 1984). In provincia di Sondrio una coppia con due pulli nel 2012 a Forcola (P. Trotti, com. pers.). In provincia di Milano nidificante in tre località: Nosate, Trezzo sull'Adda e Vasche dell'Arnetta; in provincia di Lodi singole coppie nidificanti lungo l'Adda a nord del ponte di Bisnate ed a Lodi (M. Siliprandi com., pers.). In provincia di Brescia prime nidificazioni di 2 coppie sul basso Lago di Garda nel 1986 (Cambi, 1987) e sul Lago d'Iseo nel 1991 (Mazzotti S. \& Mazzotti F, 1992), mentre nel 2001 venivano stimate circa 20 coppie, di cui oltre 10 nelle torbiere (P. Brichetti, oss. pers.). Nella parte bresciana del Lago d'Iseo stimate attualmente 10-15 coppie, alle quali vanno aggiunte circa 15 coppie presenti nella Riserva Naturale Torbiere del Sebino (L. Longo, com. pers.); per l'intero bacino lacustre, considerando le coppie presenti in provincia di Bergamo (Sarnico, Castro, Tavernola bergamasca, Costa Volpino ecc.: (P. Trotti, com. pers.) possono essere stimate 20-25 coppie. Sul Lago di Garda la popolazione attualmente presente in periodo riproduttivo può essere stimata nell'ordine di varie decine di individui, anche se le coppie realmente nidificanti non dovrebbero superare le 15-25 (oss. pers.). Nel resto della provincia di Brescia negli ultimi anni si sono verificate nidificazioni saltuarie o tentativi di singole coppie, come in una cava rinaturalizzata tra Manerbio e Leno (oss. pers.), presso Castel Mella nel 2010 (C. Chiari, com. pers.) e presso Esine nella media Val Camonica (P. Trotti, com. pers.). In provincia di Pavia una coppia immessa ha nidificato con successo nel 2004 nel Parco Palustre di Lungavilla; nello spesso Parco la specie si era riprodotta anche in anni precedenti, ma sempre con soggetti di origine "domestica"; nel 2011 una coppia di origine non nota ha nidificato con successo in un complesso di cave in località San Gaudenzio di Cervesina (F. Ferlini, com. pers.; E. Tiso com. pers.); a Pavia introdotto nel laghetto del Parco della Vernavola, con alcuni tentativi di nidificazione, di cui uno riuscito nel maggio 1997 (Bernini et al., 1998; G. Conca, com. pers.). In provincia di Mantova prima nidificazione di una coppia nel 1984 presso Castellaro Lagusello (Brichetti et al., 1984); nel 2000 accertate 16 coppie tra certe e probabili nelle Valli del Mincio-Lago Superiore e 4 coppie lungo il corso dell'Alto Mincio (Maffezzoli \& Grattini, 2002); nel 2010 la specie è stata rilevata come nidificante in 9 siti con 23 coppie certe e probabili, di cui il $69 \%$ nidificanti in due siti: Lago Superiore e R.N. Valli del Mincio (16 coppie), mentre le restanti coppie si sono riprodotte nelle R.N. Paludi di Ostiglia (1), lungo il corso del medio Mincio (2), lungo il corso del Basso Mincio presso Formigosa (1), in cave artificiali marginali nei pressi di Bosco della Fontana in comune di Marmirolo e Goito (3) (Grattini et al., 2013); successivamente una coppia nel 2011-12 presso Chiavica del Moro lungo il Basso Mincio (oss. pers.), una coppia con pulli sul Lago Inferiore nel 2012, una coppia con pulli nel 2012 presso Volta Mantovana (A. Peri, com. pers.); nel giugno 2012 presenti 51 individui non nidificanti presso la R.N. Vallazza (S. Bellintani, com. pers).

Trentino-Alto Adige: in provincia di Trento nidificante dal 1989 dopo introduzioni a fini ornamentali presso Riva del Garda; nel 1994 una coppia ha nidificato nel bacino di Ponte Pià; nidificante dai primi anni '90 nel Lago di Caldonazzo con 1-2 coppie e con una coppia nel Lago di Serraia nel 1995 (Caldonazzi et al., 1997); una coppia ha nidificato presso la Chiusa di Mori sull'Adige almeno sino al 2000. La popolazione provinciale è valutata in 5-8 coppie (Pedrini et al., 2005). In provincia di Bolzano una coppia nidificante al lago di Caldaro e nel 1990 un caso di nidificazione presso il Rio Pusteria (Niederfriniger, 1998). Nel 2011-12 una coppia si è riprodotta con certezza sul Lago di Dobbiaco, con osservazioni in periodo di nidificazione a Brunico e Bressanone, mentre le coppie presenti negli ultimi anni sul Lago di Caldaro non sembrano più riprodursi per disturbo antropico $(\mathrm{O}$. Niederfriniger, com. pers.).

Veneto: in provincia di Verona almeno due coppie si sono riprodotte dal 1987 al 1989 presso Peschiera del Garda (Brichetti et al., 1992); una coppia nel 2002 nel Laghetto del Frassino (Morbioli \& Sighele, 2006); singole coppie in cova sul Mincio a Borghetto e Valeggio nel 2011-2012 (G. Dalle Vedove, com. pers.). Nella parte veneta del Lago di Garda stimate negli ultimi anni circa 10 coppie tra Pescheria e Malcesine (M. Sighele, com. pers.). In provincia di Venezia stimate 150-200 coppie (Bon et al., 2000); nella stessa provincia un'indagine condotta nel 2003 ha evidenziato la presenza di circa 50 coppie così distribuite: 9 coppie nel tratto fluviale Musile di Piave, Caposile, Faro di Piave Vecchia; 4 coppie nel tratto fluviale di San Michele Vecchio-Caposile; 3 coppie nel fiume Livenza e Brian; 10 coppie nella Valli Laguna nord di Venezia; 19 coppie nel Canale Nicesolo e Valli di Caorle e Bibione; 7 coppie a Boldara, Ceggia, Musile di Piave, Torre di Fine, Passerella di Sotto, Cortellazzo e Revedoli (Cappelletto \& Zanetti, 2004).

In provincia di Padova nidificazioni accertate in Valle Morosina (2 coppie) e nel centro di Padova nel periodo 1992-1994 in condizioni di quale totale domesticità (Nisoria, 1997); successivamente almeno 2 coppie nidificanti a Piazzola sul Brenta (G. Piras, com. pers.), 1-2 coppie dal 2002 al 2012 tra Camposampiero-Campodarsego e altrettante o forse più nei canali di Padova in località Bassanello (S. Bottazzo, com. pers.); nel medio corso del Brenta 6 nidificazioni accertate e 4 possibili nel periodo 2001-2008, con primi dati nel 2001, escludendo una nidificazione del 1993 in regime di semidomesticità (Bonato \& Farronato, 2012). Nel Delta del Po Veneto (RO) una coppia nidificante dal 2000 in Valle Sacchetta (Fracasso, 2003), dove a seguito di un progressivo incremento la popolazione è stimata in 15-25 coppie nel periodo 1992-2002 (Verza \& Bottazzo, 2011). In provincia di Belluno il bacino artifi- 
ciale di Busche, lungo l'asta idrica del Piave, ospita l'unico nucleo nidificante con certezza (almeno 2 coppie) e rappresenta il punto di irradiamento di soggetti immessi presumibilmente nei primi anni '70; sempre lungo il Piave una coppia si riproduce poco più a monte, mentre altrove sono note presenze in periodo riproduttivo presso $\mathrm{Mel}$ e in località Marzini senza prove di nidificazione, come lungo il torrente Cismon presso Arten; coppie immesse sono presenti in alcuni laghetti di pesca sportiva, come a Ponte delle Alpi (A. De Faveri, com. pers.). In provincia di Vicenza almeno una coppia nidificante nel Lago di Fimon negli ultimi anni (Cerato \& Fracasso, 2014).

Friuli-Venezia Giulia: in provincia di Udine nidificante regolarmente con 1-2 coppie dal 1988 in Valle Canal Novo (Vicario, 2003); nidificazioni saltuarie dal 1982 alle Foci dello Stella e dal 1984 nella Sacca di Muzzana (Guzzon, 2003); in Valle Cavanata prime nidificazioni con successo nel 1985-87 (Utmar, 1989); in provincia di Gorizia alle foci dell'Isonzo dall'inizio degli anni 2000 si è assistito ad un notevole aumento di coppie nidificanti, fino ad un max. di 12 (Perco et al., 2006); in provincia di Gorizia nidificante dal 1982 con una coppia in Valle Cavanata; nel 1997 e nel 1998 una coppia nel Canale Quarantia (Foce dell'Isonzo); mentre nel 1995 hanno nidificato 9 coppie (Parodi, 1999): nella Cassa di Colmata del Lisert, presso Monfalcone dal 2004 con una coppia sino a 7-8 coppie nel 2010 (Utmar, 2011). In uno studio effettuato nel 2005-07 nelle zone costiere regionali sono state censite 45 coppie nel 2005, 63 nel 2006 e 61 nel 2007, così distribuite: Lisert 2-3 coppie; foce Isonzo 8-17 coppie; Valle Cavanata 17-21 coppie; laguna di Grado 6-12 coppie; laguna di Marano 8-18 coppie (Ventolini, 2008). La consistenza delle popolazioni nidificanti in regione nel periodo 2008-2012 è valutata in 101-129 coppie, con nuclei più consistenti (>3 coppie) a Foci Timavo Lisert (7-9), Foce Isonzo (45), Isola della Cona (8-10), Valle Cavanata (18-21), Valle Panera (3-5), Valle Noghera (4), Valle Pantani (3-5), Foci Stella (21-24), Sacca di Muzzana (7-9) (C. Guzzon, K. Kravos, P. Utmar, com. pers.).

Emilia-Romagna: in provincia di Piacenza nel 2000 una coppia ha nidificato nell'Oasi di Pinedo (Ambrogio et al., 2001) e attualmente la presenza è probabile anche in altre località. In provincia di Reggio nell'Emilia nidificante una coppia dal 2010 a Cà de Frati in comune di Rio Saliceto (L. Bagni e N. Grattini, oss. pers.). In provincia di Modena la popolazione, originatasi da una coppia immessa nelle vasche della Tomina (Valli del Mortizzuolo), non supera attualmente le 5-6 coppie, sostanzialmente stabili negli ultimi 10 anni (C. Giannella, com. pers.). In provincia di Bologna nidificante presso Valle La Boscosa (Molinella), dove 2 coppie introdotte nel 1997 si sono riprodotte con successo nel 1999, mentre nel 2001 una coppia si è insediata spontaneamente ed ha nidificato con successo presso Baricella (Tinarelli et al., 2002); nella stessa provincia le coppie nidificanti negli ultimi anni variano tra 6 e 10, escludendo gli individui tarpati di vari laghetti che producono ogni anno giovani che si irradiano nel territorio circostante (R. Tinarelli, com. pers.). In provincia di Ferrara nidificazioni dagli anni '80 in zone umi- de dell'interno a seguito di immissioni (Valli di Argenta, Anse di Ostellato, Vallette di Portomaggiore), oltre che nell'area del Delta del Po e delle Valli di Comacchio (oss. pers.; A. Talamelli, com. pers.); altrove 2-4 coppie rilevate in Valle Bertuzzi nel 2005-2006 (Costa et al., 2009). In provincia di Ravenna prime nidificazioni accertate di 1-2 coppie nella Pialassa Baiona nel 2004, aumentate a 3-5 nel 2006; in Valle Mandriole 1-2 coppie nel 1995 e 2-3 nel 2006; nell'Ortazzo 1 coppia nel 2004 (Gellini \& Ceccarelli, 2011). Nella parte regionale del Parco del Delta del Po la popolazione è incrementata da 6-7 coppie nel 2004 a 8-11 nel 2006 (Costa et al., 2009;).

In provincia di Rimini 1 coppia ha nidificato nel 2006 in un piccolo stagno situato tra Riccione e Misano Adriatico (Casini \& Gellini, 2008), dove nidifica tuttora (A. Talamelli, com. pers.).

Toscana: una coppia nidificante dal 1994 nel Lago di Burano (GR) e una osservata nel maggio-giugno 1986 nelle vasche dello zuccherificio di Castiglion Fiorentino (AR) (Tellini et al., 1997). A Firenze 2 coppie nidificanti allo stato semi-domestico nella seconda metà degli anni '80 (Dinetti \& Ascani, 1990); negli ultimi anni, escludendo segnalazioni primaverili senza indizi di nidificazione, 1 coppia si riproduce irregolarmente allo stato naturale nel lago di Burano, dove la specie risulta introdotta da alcuni decenni (L. Puglisi, com. pers.); in Toscana settentrionale recente nidificazione di individui di origine domestica in un laghetto del Golf Club Versilia (E. Arcamone, com. pers.).

Marche: in provincia di Macerata nel 2012 una coppia nidificante sul fiume tra Pioraco e Sefro nel 2012, probabilmente immessa dal comune di Pioraco (P. Forconi, com. pers.).

Umbria: in provincia di Terni nidificante regolarmente da qualche anno al Lago di Alviano con 4-5 coppie; diversi individui sostano per periodi variabili anche sul Lago di Recentino, con tentativo di nidificazione circa 20 anni fa, e sul Lago di S. Liberato, dove attualmente non nidifica ma nel 2012 è stata presente 1 coppia territoriale (S. Laurenti, com. pers.).

Lazio: in provincia di Latina 1 coppia sui Laghi Pontini (F. Corbi, oss. pers.); in provincia di Rieti 1 coppia in condizioni seminaturali nei Laghi Reatini (M. Brunelli, com. pers.; Brunelli et al., 2011); in provincia di Roma almeno 5 coppie sul Lago di Bracciano (M. Bernoni, oss. pers.) e 2 coppie sul Litorale Romano (M. Biondi, oss. pers.); in provincia di Viterbo 3-5 coppie sul Lago di Bolsena (E. Calvario, oss. pers.).

Basilicata: prima nidificazione accertata nella regione di 1 coppia nel luglio 2004 in provincia di Potenza presso il Lago Pantano di Pignola (Guardascione, 2005), dove da allora si riproduce regolarmente (E. Fulco e M. Visceglia, com. pers.); 1 coppia si riproduce irregolarmente sul Laghi di Monticchio-M. Vulture, dove sono presenti molti altri soggetti non nidificanti; si tratta in entrambi i casi di piccole popolazioni originatesi da immissioni avvenute negli anni '90 (E. Fulco, com. pers.). 
Puglia: tentativo di nidificazione nel Salento a inizio anni '90 (Nicolì, 1993); il Cigno reale attualmente non sembra nidificare nelle aree naturali pugliesi dove è solo migratore e svernante irregolare (G. La Gioia e M. Marrese, com. pers.); non si esclude la presenza di qualche coppia domestica in giardini privati (G. La Gioia, com. pers.).

Sardegna: prima introduzione nell'isola effettuata intorno al 1955 in un piccolo lago artificiale della parte centro-occidentale, presso Villagrande (NU), con rilascio di 1 coppia di adulti importata dalla Svizzera, nidificante con successo; attorno al 1990 una piccolissima popolazione è stata introdotta in alcuni siti della parte nord-orientale dell'isola, in Costa Smeralda; per diversi anni, 1-2 coppie si sono anche insediate e riprodotte nella vicina località di Liscia di Vacca; attualmente 2-3 coppie tenute in condizioni di semilibertà nidificano ancora in Costa Smeralda (Sanna, inedito). Nello stesso periodo la riproduzione si è verificata nel Golfo di Cagliari all'interno del parco di un hotel presso Pula (Grussu, 1995). Inoltre, sempre in quest'area, in località Poggio dei Pini, tra la fine del XX secolo e i primi del XXI, alcune coppie sono state rilasciate in zone umide artificiali, dove nel periodo 2006-2008 una coppia nidifica regolarmente. In totale, in Sardegna attualmente sono presenti almeno 3-4 coppie nidificanti su una popolazione di 20-40 individui (Grussu, 2008). Inoltre nel 2010-11 singole coppie hanno nuovamente nidificato allo stato semidomestico nel Golfo di Cagliari, ma con scarsi risultati riproduttivi a causa della predazione di Cornacchia grigia, Corvus cornix, (M. Grussu, com. pers.; Gruppo Ornitologico Sardo).

\section{CONCLUSIONI}

L'attuale areale di nidificazione primario risulta concentrato nelle regioni settentrionali, in corrispondenza dei laghi insubrici e pedemontani, tra Piemonte orientale e Veneto settentrionale, oltre che nella fascia costiera altoadriatica, tra il Ferrarese e il Goriziano; in quest'area si riproduce circa il $90 \%$ della popolazione nazionale. Presenze più frammentate $\mathrm{e} / \mathrm{o}$ instabili, in gran parte dovute $\mathrm{a}$ immissioni più o meno recenti, in zone interne della Pianura Padana, soprattutto in corrispondenza dei principali corsi d'acqua, oltre che in Liguria, Trentino-Alto Adige, Toscana, Umbria, Lazio, Basilicata e Sardegna.

La colonizzazione di molti siti, ubicati prevalentemente nelle regioni settentrionali, è conseguente alla presenza ripetuta di nuclei svernanti, cui ha fatto seguito la formazione di coppie riproduttrici; tale fenomeno ha interessato, seppur in minor misura anche le regioni centro-meridionali, dove le presenze in periodo extrariproduttivo sono andate aumentando nel corso degli ultimi due decenni.

La popolazione nazionale nell'arco di circa 30 anni ha fatto registrare sia un progressivo incremento numerico sia una vistosa espansione di areale a sud del corso del Po, più sensibilmente a partire da metà/fine anni ' 90 , passando dalle 20-35 di inizio anni ' 80 del secolo scorso, presenti in meno di 15 siti, alle 500-700 coppie degli ultimi anni, distribuite in più di 150 siti con un aumento di oltre il $900 \%$, a conferma della fase di incremento ed espansione territoriale rilevata a livello europeo negli ultimi decenni.

\section{Ringraziamenti}

Desideriamo ringraziare per le informazioni e dati inediti forniti: Emiliano Arcamone, Luca Bagni, Simone Balbo, Banca Dati della SSNVCO, Stefano Bellintani, Enrico Benussi, Mauro Bernoni, Radames Bionda, Massimo Biondi, Lucio Bordignon, Stefano Bottazzo, Enrico Bresciani, Massimo Brunelli, Enrico Calvario, Monica Carabella, Carlo Chiari, Gianni Conca, Ferdinando Corbi, Giangaetano Dalle Vedove, Adriano De Faveri, Clemente Fabrizio, Flavio Ferlini, Paolo Forconi, Egidio Fulco, Carlo Giannella, Gruppo Ornitologico Sardo, Carlo Guzzon, Marcello Grussu, Kajetan Kravos, Giuseppe La Gioia, Stefano Laurenti, Luca Longo, Daniele Longhi, Maurizio Marrese, Alessandro Micheli, Oskar Niederfriniger, Francesco Ornaghi, Menotti Passarella, Achille Peri, Luca Puglisi, Maurizio Sighele, Marco Siliprandi, Adriano Talamelli, Roberto Tinarelli, Eugenio Tiso, Aldo Tonelli, Paolo Trotti, Rudy Valfiorito, Francesco Velatta, Enrico Vigano, Matteo Visceglia, Stefano Volponi, Michele Zanetti.

\section{PUBBLICAZIONI CITATE}

Ambrogio A. Figoli G. \& Ziotti L. (eds.), 2001 - Atlante degli uccelli nidificanti nel Piacentino. LIPU Sezione di Piacenza.

Bernini F. Dinetti M. Gariboldi A. Matessi G. \& Rognoni G. (eds.), 1998 - Atlante degli uccelli nidificanti a Pavia. Comune di Pavia.

Bionda R. \& Bordignon L. (eds.), 2006 - Atlante degli uccelli nidificanti nel Verbano Cusio Ossola. Quaderno Natura e Paesaggio del VCO N. 6.

BirdLife International, 2004 - Birds in Europe: population estimates, trends and conservation status. Cambridge: BirdLife International.

Boano G. \& Brichetti P. 1989 - Proposta per una classificazione corologica dell'avifauna italiana. I. Non Passeriformi. Rivista italiana di Ornitologia, 59: 141158.

Bonato R. Farronato I. 2012 - Uccelli del medio corso del fiume Brenta. Una fenologia. Parte prima. Gruppo di Studi Naturalistici "Nisoria". www.faunistiveneti.it

Bon M. Cherubini G. Semenzato S. \& Stival E. (eds.), 2000 - Atlante degli uccelli nidificanti in provincia di Venezia. Provincia di Venezia.

Bordignon L. \& Lonati S. (eds.), 2011 - Avifauna Valsesiana, 25 anni di storia. Borgomanero: Litpress.

Borgo E. Galli L., Galuppo C. Maranini N. \& Spanò S. (eds.), 2005 - Atlante ornitologico della Città di Genova (1996-2000). Boll. Musei e Ist. Biologici Univ. di Genova, Vol. 69-70.

Brichetti P. Canova L. \& Saino N, 1984 - Distribuzione e status degli Anatidae nidificanti in Italia e Corsica. Avocetta 8: 19-42.

Brichetti P. \& Fasola M. (eds.), 1990 - Atlante degli uccelli nidificanti in Lombardia 1983-1987. Brescia: Editoriale Ramperto. 
Brichetti P. \& Gariboldi A. 1992 - Manuale pratico di ornitologia Vol. 2. Bologna: Edagricole.

Brichetti P. \& Fracasso G. 2003 - Ornitologia italiana. Vol. I. Gaviidae-Falconidae. Bologna: Alberto Perdisa Editore.

Brichetti P., 2012 - Banca Dati Ornitologica - BDO. Bibliografia italiana, 1900-2012. Versione elettronica.

Brunelli M. Sarrocco S. Corbi F., Sorace A. Boano A. De Felici S. Guerrieri G. Meschini A. \& Roma S. (eds.), 2011 - Nuovo Atlante degli Uccelli Nidificanti nel Lazio. Edizioni Agenzia Regionale Parchi, Roma.

Caldonazzi M., Pedrini P. \& Zanghellini S, 1997 - Il Monitoraggio sugli uccelli acquatici nei biotopi del Trentino. Collana del Piano di valorizzazione didattica, culturale, ricreativa e sociale dei biotopi tutelati. Provincia Autonoma di Trento. Serie bianca: Documenti.

Cambi D., 1987 - Ulteriori dati ornitologici dal Garda. Rivista italiana di Ornitologia 57: 117-120.

Cappelletto M. \& Zanetti M. 2004 - Il Cigno reale nelle lagune e nei fiumi del Veneto orientale. Associazione Naturalistica Sandonatese.

Casini L. \& Gellini G. (eds), 2008 - Atlante dei Vertebrati tetrapodi della provincia di Rimini. Provincia di Rimini.

Caula B. Beraudo P.L. \& Toffoli R. (eds.), 2005 - Gli Uccelli della provincia di Cuneo. Check list commentata delle specie. Lab. Terr. Educ. Amb. Museo Civico Craveri di Storia Naturale, Brà.

Cerato E. \& Fracasso G. 2014 - Uccelli dei Colli Berici. Provincia di Vicenza.

Costa M. Ceccarelli P.P. Gellini S. Casini L. \& Volponi S. (eds.), 2009 - Atlante degli uccelli nidificanti nel Parco del Delta del Po (Emilia-Romagna (2004-2006). Parco Delta del Po Emilia-Romagna.

Cramp S. \& Simmons K.E.L. (eds.), 1977 - The Birds of the Western Palearctic. Vol. I. Oxford: Oxford University Press.

Dinetti M. \& Ascani P. (eds.), 1990 - Atlante degli uccelli nidificanti nel Comune di Firenze. Comune di Firenze.

Fracasso G. Baccetti N. \& Serra L, 2009 - La lista CISOCOI degli Uccelli italiani - Parte prima: liste A, B e C. Avocetta, 33: 5-24

Fracasso G. Verza E. \& Boschetti E. (eds.), 2003 - Atlante degli uccelli nidificanti in provincia di Rovigo. Provincia di Rovigo.

Gagliardi A. Guenzani W. Preatoni D.G. Saporetti F. \& Tosi G. (eds.), 2007 - Atlante Ornitologico Georeferenziato della provincia di Varese - Uccelli nidificanti 2003-2005. Prov. di Varese, Museo Insubrico St. Nat. di Induno Olona e Univ. dell'Insubria di Varese.

Gellini S. \& Ceccarelli P.P. (eds.), 2011 - Atlante degli uccelli nidificanti nelle province di Forlì-Cesena e Ravenna. (2004-2007). S.T.E.R.N.A., Forlì.

G.P.S.O. (red. Maffei G. \& Della Toffola M.), 1992 - Resoconto ornitologico per la regione Piemonte e Valle d'Aosta. Anno 1991. Riv. Piem. St. Nat., 13: 103122.

Grattini N. Longhi D. \& Peri A, 2013 - Distribuzione e consistenza di cigno reale, Cygnus olor, svasso maggiore, Podiceps cristatus e folaga, Fulica atra, nidificanti in provincia di Mantova. Pianura, 30: 101-107.
Gruppo Nisoria, (eds.), 1997 - Atlante degli uccelli nidificanti nella provincia di Padova. Vicenza: Gilberto Padovan Editore.

Grussu M., 1995 - Status, distribuzione e popolazione degli uccelli nidificanti in Sardegna (Italia) al 1995 (Prima parte). Uccelli d'Italia 20: 77-85.

Grussu M., Basoccu P., Sanna M. \& Mascia F., 2008 - La popolazione alloctona di cigno reale (Cygnus olor) in Sardegna On the introduced population of Mute Swan (Cygnus olor) in Sardinia - Le specie alloctone in Italia: censimenti, invasività e piani di azione. Memorie della Società Italiana di Scienze Naturali e del Museo Civico di Storia Naturale di Milano. Volume XXXVI, Fasc. I.

Guardascione E., 2005 - Nidificazione di Cigno reale, Cygnus olor, in Basilicata, Lago Pantano di Pignola, provincia di Potenza. Rivista italiana di Ornitologia, 75: 58-59.

Guenzani W. \& Saporetti F. (eds.), 1988 - Atlante degli uccelli nidificanti in Provincia di Varese (Lombardia) 1983-1987. LIPU Sezioni varesine. Varese.

Guzzon C., 2003 - Monitoraggio ornitologico e checklist delle specie della Riserva Naturale Regionale delle Foci dello Stella, Marano Lagunare 1998-2002. Regione Autonoma Friuli-Venezia Giulia.

Hagemeijer WJM. \& Blair MJ., 1997 - The EBCC Atlas of European breeding birds. London: T. e AD Poyser.

LIPU e WWF., 1999 - Nuova Lista Rossa degli Uccelli nidificanti in Italia. Rivista italiana di Ornitologia, 69: 3-43.

Maffei G., Pulcher C., Rolando A. \& Carisio L. (eds.), 2001 - L'avifauna della città di Torino: analisi ecologica e faunistica. Monografie Mus. reg. Sci. nat. Torino XXXI.

Maffezzoli L. \& Grattini N., 2002 - Distribuzione e consistenza del Cigno reale, Cygnus olor, nidificante in Provincia di Mantova. Rivista italiana di Ornitologia, 71: 209-211

Mazzotti S. \& Mazzotti F., 1992 - Prima nidificazione allo stato selvatico del Cigno reale Cygnus olor sul Lago d'Iseo (Brescia). Natura Bresciana, 27: 311.

Mingozzi T., Boano G., Pulcher C. \& coll., 1988 - Atlante degli uccelli nidificanti in Piemonte e Val d'Aosta 1980-1984. Monografie VIII, Museo Regionale di Scienze Naturali di Torino.

Morbioli M. \& Sighele M., 2006 - L'avifauna del Laghetto del Frassino (Peschiera del Garda, Verona, Veneto) - Bollettino del Museo Civico di Storia Naturale di Verona 30, Botanica Zoologia: 275-291.

Nicolì A., 1993 - Tentativo di nidificazione del Cigno reale nel Salento. Migratori alati 16/3-4: 34

Parodi R. \& Perco F., 1992 - Cigno reale Cygnus olor (pp. 246-252). In: Brichetti P., De Franceschi P. \& Baccetti N. (a cura di). Fauna d'Italia, XXIX, Aves. I. Bologna: Calderini.

Niederfriniger O., Schreiner P. \& Unterholzner L. (eds.), 1998 - Atlante dell'avifauna dell'Alto Adige. AVK. Bolzano: Tappeiner/Athesia.

Parodi R. (ed.), 1999 - Gli uccelli della provincia di Gorizia. Museo Friulano Storia Naturale Pubbl. n. $42 \mathrm{Mu}-$ seo Friulano di Storia Naturale, Udine. 
Pedrini P., Caldonazzi M. \& Zanghellini S. (eds.), 2005 Atlante degli Uccelli nidificanti e svernanti in provincia di Trento - Suppl. Acta Biologica 80 (2003). Museo Tridentino di Scienze Naturali.

Perco F., 1988 - Problemi di conservazione e gestione degli Anseriformi in Italia. Suppl. Ric. Biol. Selvaggina 14: $19-50$.

Perco F., Merluzzi P. \&. Kravos K, 2006 - La Foce dell'Isonzo e l'Isola della Cona. Mariano del Friuli (GO): Edizioni della Laguna.

Peronace V., Cecere J. G., Gustin M. \& Rondinini C. (eds.), 2012 - Lista Rossa 2011 degli Uccelli Nidificanti in Italia. Avocetta, 36: 11-58.

Tellini F. G., Baccetti N., Arcamone E., Meschini E. \& Sposimo P. (eds.), 1997 - Atlante degli uccelli nidificanti e svernanti in Toscana (1982-1992). Quaderni del Museo Provinciale di Storia Naturale di Livorno, Monografie 1.

Tinarelli R., Bonora M. \& Balugani M. (eds.), 2002 - Atlante degli Uccelli nidificanti nella Provincia di Bologna (1995-1999). Comitato per il Progetto Atlante Uccelli Nidificanti nella Provincia di Bologna. Su CDROM.

Utmar P., 1989 - Gli anatidi nidificanti nella provincia di Gorizia e nella laguna di Marano. Fauna 1: 32-46.

Utmar P., 2011 - Nidificazione di Sterna comune, Sterna hirundo, in nidi abbandonati di Cigno reale, Cygnus olor, nella Cassa di Colmata del Lisert, Monfalcone (GO) e importanza ornitologica del sito. Suppl. Bollettino Museo di Storia Naturale di Venezia, vol. 61.

Ventolini N., 2008 - La popolazione di Cigno reale nella zona costiera del Friuli-Venezia Giulia. Tesi di dottorato di ricerca, Università di Trieste.

Verza E. \& Bottazzo M., 2011 - Le anatre selvatiche del Delta. Monitoraggi e ricerche sugli Anatidi nel Delta del Po (Veneto). Veneto Agricoltura.

Vicario G., 2003 - Monitoraggio ornitologico e checklist delle specie della Riserva Naturale Regionale della Valle Canal Novo, Marano Lagunare 1997-2002. Regione Autonoma Friuli-Venezia Giulia.

Vigorita V. \& Cucè L., 2008 - La fauna selvatica in Lombardia. Rapporto 2008 su distribuzione, abbondanza e stato di conservazione di uccelli e mammiferi. Regione Lombardia. 